\title{
Impfung reduziert Postzosterneuralgien
}

Fragestellung: Kann durch eine spezifische Impfung bei Menschen über 70 Jahren das Auftreten von Herpes zoster und einer Postzosterneuralgie verhindern?

Hintergrund: Der Herpes zoster ist eine typische Alterserkrankung mit einer Inzidenz von etwa elf pro 1.000 über 80-Jährigen. Der Herpes zoster selbst kann heute sehr gut behandelt werden. Die virustatische Therapie hat jedoch keinen Einfluss auf das Auftreten einer chronischen Postzosterneuralgie. Ein spezifischer Impfstoff gegen das Varizella-zoster-Virus wurde zunächst bei Menschen im Alter über 50 Jahren untersucht und als wirksam identifiziert [1]. Jetzt sollte eine Studie bei über 70-Jährigen durchgeführt werden.

Cunningham AL, Lal H, Kovac $M$ et al. Efficacy of the herpes zoster subunit vaccine in adults 70 years of age or older. $N$ Engl $J$ Med 2016; 375: 1019-32
Patienten und Methodik: Die randomisierte placebokontrollierte Studie wurde in 18 Ländern durchgeführt. Die Teilnehmer waren älter als 70
Jahre und erhielten entweder zwei Dosierungen des Impfstoffs oder Placebo. Die Injektion erfolgte intramuskulär im Abstand von zwei Monaten. Erfasst wurde das Auftreten von Herpes zoster und postherpetischen Neuralgien.

Ergebnisse: In die Studie wurden 13.900 Teilnehmer aufgenommen. Das mittlere Alter betrug 75,6 Jahre. Während der 3,7-jährigen Beobachtungszeit gab es 23 Herpes-zoster-Fälle in der Impf- und 223 in der Placebogruppe. Dies entspricht einer Reduktion von $90 \%$. Der Effekt war im Alter zwischen 70 und 79 Jahren und bei über 80 -Jährigen gleich. Wurden diese Ergebnisse mit den Resultaten der bei 50-Jährigen durchgeführten Studie kombiniert, ergab sich eine Reduktion der Herpes-zoster-Fälle um $91 \%$ und der Postzosterneuralgien um $88 \%$.

Schlussfolgerung: Ein spezifischer Impfstoff gegen Varizella zoster kann bei Menschen im Alter über 50 Jahren hochsignifikant das Auftreten eines Herpes zoster und einer Postzosterneuralgie verhindern.

\section{- Kommentar von Hans-Christoph Diener, Essen}

\section{Wichtiger Aspekt für die Beratung älterer Patienten}

Diese Studie ist sehr wichtig für die Beratung älterer Menschen, insbesondere, wenn sie immunsupprimiert sind oder ein erhöhtes Infektionsrisiko aufweisen. Dies gilt unter anderem auch für Patienten mit Diabetes mellitus. Für Neurologen ist besonders relevant, dass nicht nur das Auftreten der Herpes-zoster-Erkrankung als solche hochsignifikant reduziert wird, sondern vor allem, dass seltener postzosterische Neuralgien auftreten. Jeder Kliniker weiß, wie schwer es ist, bei al- ten Menschen eine Postzosterneuralgie zu behandeln, da diese Patientenpopulation auf die meisten der verfügbaren Medikamente bezüglich Nebenwirkungen sehr ungünstig reagiert.

\author{
Referenz: \\ 1. Lal H et al. N Engl J Med 2015; 372: 2087 - 96
}

\section{Ketamininfusion ein- oder zweimal pro Woche?}

Fragestellung: Gibt es Unterschiede in der antidepressiven Wirksamkeit von Ketamin bei zwei- oder dreimal wöchentlicher intravenöser Applikation bei therapieresistenter Depression (TRD)?

Hintergrund: Ketamin ist ein nicht kompetitiver, N-methyl-DAspartat-Glutamatrezeptorantagonist, der als Anästhetikum zugelassen ist. In neuesten Studien wurde zudem über einen schnell einsetzenden antidepressiven Effekt durch Ketamin berichtet, der jedoch nicht lange anhaltend war (zwischen drei und 17 Tagen). Überwiegend wurde in diesen Studien eine einmalige Ketamininfusion gegeben. In Studien, in denen insgesamt sechs Ketamininfusionen verabreicht wurden, zeigte sich ein anhaltender antidepressiver Effekt über sieben bis 28 Tage. Bisher ist nicht bekannt, wie dieser antidepressive Effekt etwa durch ein längerfristiges Dosierungsschema aufrechterhalten werden kann. Das primäre Ziel dieser Studie war es, den Verlauf der antidepressiven Wirksamkeit von zwei in der Frequenz unterschiedlichen Applikationsschemata bei Ketamininfusionen (zwei- vs. dreimal wöchentlich) im Vergleich zu Placebo zu untersuchen.

Patienten und Methodik: Die doppelblinde, randomisierte, placebokontrollierte Phase-II-Studie mit Parallelgruppendesign 